\title{
Downregulation of hsa_circ_0026123 suppresses ovarian cancer cell metastasis and proliferation through the miR-124-3p/EZH2 signaling pathway
}

\author{
XIAOYUN YANG ${ }^{1}$, JIANJUN WANG $^{1}$, HUAIFANG LI ${ }^{1}$, YI SUN ${ }^{2}$ and XIAOWEN TONG ${ }^{1}$ \\ ${ }^{1}$ Department of Gynecology and Obstetrics, Tongji Hospital, Tongji University School of Medicine, Shanghai 200065; \\ ${ }^{2}$ Department of Gynecology and Obstetrics, Tongren Hospital, Shanghai Jiaotong University \\ School of Medicine, Shanghai 200336, P.R. China
}

Received May 4, 2020; Accepted October 8, 2020

DOI: $10.3892 /$ ijmm.2020.4804

\begin{abstract}
Circular RNAs (circRNAs) play a role in various types of cancer. The present study suggested that hsa circ_0026123 expression was upregulated in ovarian cancer (OVA), which was associated with its role in OVA. However, the role of hsa_circ_0026123 in OVA cell invasion and proliferation remains unclear. In the present study, OVA tissues and cell lines were used to investigate the functions of hsa_circ_0026123. The associations between hsa_circ_0026123, miR-124-3p and enhancer of zeste homolog 2 (EZH2) were examined using a luciferase reporter assay. RT-qPCR and western blot analysis were used for gene and protein expression analysis, respectively. Tumor growth was detected using nude mouse tumor xenografts derived from SKOV3 cells, with or without hsa_circ_0026123 downregulation. The results confirmed that hsa_circ_0026123 expression was upregulated in OVA tissues and cell lines, while hsa_circ_0026123 silencing suppressed cell proliferation and migration; it also suppressed the expression of cancer stem cell (CSC) differentiation-related markers in either in vivo or in vitro experiments. The data revealed that hsa_circ_0026123 downregulation suppressed EZH2 expression by miR-124-3p 'sponging', which was confirmed
\end{abstract}

Correspondence to: Dr Yi Sun, Department of Gynecology and Obstetrics, Tongren Hospital, Shanghai Jiaotong University School of Medicine, 1111 Xianxia Road, Shanghai 200336, P.R. China E-mail: sunyijojo@163.com

Dr Xiaowen Tong, Department of Gynecology and Obstetrics, Tongji Hospital, Tongji University School of Medicine, 389 Xincun Road, Putuo, Shanghai 200065, P.R. China

E-mail: xiaowen_tong@hotmail.com

Abbreviations: OVA, ovarian cancer; circRNAs, circular RNAs; miRNA/miR, microRNA; FBS, fetal bovine serum; siRNA, small interfering RNA; NC, negative control; CSC, cancer stem cell

Key words: hsa_circ_0026123, ovarian cancer, miR-124-3p, enhancer of zeste homolog 2 , cancer stem cell markers by rescue experiments and luciferase reporter assays. The results revealed that hsa_circ_0026123 silencing suppressed ovarian cancer cell progression via the miR-124-3p/EZH2 signaling pathway. Overall, the findings demonstrated that hsa_circ_0026123 knockdown inhibited OVA cell progression by regulating the miR-124-3p/EZH2 axis. This methodology may thus be used for the targeted therapy of OVA, as well as a candidate biomarker for the diagnosis and treatment of OVA.

\section{Introduction}

Ovarian cancer (OVA) is a common malignancy of the female reproductive system, and is associated with the highest mortality rate of all gynecological malignant diseases (1). Although advancements have been made in surgical treatments and chemotherapy, the 5-year survival of patients with OVA is only approximately $30 \%$ due to frequent recurrence $(2,3)$. Thus, a main objective for the treatment of OVA is the identification of novel prognostic biomarkers, which can be used to distinguish patients at a high risk of relapse and to detect biomarkers that are candidate therapeutic targets. Previous studies have suggested that circular RNAs (circRNAs) play an important role in the progression and development of a number of types of cancer, and have promising therapeutic and prognostic value $(4,5)$. However, the molecular mechanisms of action of circRNAs in OVAs remain largely unclear.

circRNAs belong to a family of regulatory non-coding RNA (ncRNA) molecules that consist of covalently-closed and continuous loop structures without a $5^{\prime}$ cap and $3^{\prime}$ polyA tail (6). The majority of circRNAs are produced from a precursor mRNA backsplicing (7). The involvement of circRNAs in cancer pathology has been extensively investigated. It has been reported that circRNAs negatively modulate microRNA (miRNA or miR) expression by interacting with binding sites, which subsequently affects the downstream levels of mRNAs (6). A major circRNA function is to function as a miRNA sponge (8-10). The dysregulation of the circRNA/ miRNA/mRNA axis in signaling pathways plays a role in various types of cancer, including OVA (11-13). For example, circPLEKHM3 functions as a tumor suppressor via the regulation of the miR-9/DNAJB6/BRCA1/KLF4/AKT1 axis in 
OVA (14). circRNA1656 has been shown to be downregulated in high-grade serous OVA tissues and cell lines, and has been shown to be a novel biomarker (15). circRNA UBAP2 has been shown to promote OVA progression by sponging miR-144 (16).

The present study investigated the functional role of hsa_circ_0026123 and found that hsa_circ_0026123 expression was upregulated in OVA tissues and cell lines; however, hsa_circ_0026123 silencing suppressed cell proliferation and migration, and decreased the expression of markers associated with cancer stem cell (CSC) differentiation via the regulation of the miR-124-3p/enhancer of zeste homolog 2 (EZH2) signaling pathway. These results may provide a viable reference for the clinical diagnosis and treatment of OVA.

\section{Materials and methods}

Animal ethics statement. The present study used $12 \mathrm{BALB} / \mathrm{c}$ female nude mice (4 weeks old; weighing 15-20 g; SLARC). The mice were housed in a temperature-controlled $\left(25^{\circ} \mathrm{C}\right)$ room under a 12-h light/dark cycle to mimic the normal physiological the day/night cycle. Standard chow and water were freely available to the animals following sterilization. The Ethics Committee of Shanghai Tongji Hospital, Tongji University, Shanghai, China approved all animal experiments.

Tissue specimens. OVA tissues $(\mathrm{n}=20)$ and healthy fallopian tube tissue $(n=20)$ were obtained (from patients aged 48-74 years) undergoing surgery (healthy fallopian tube tissues were from the unilateral removal of fallopian tubes) at the Shanghai Tongji Hospital from January, 2019 to July, 2019 were sequentially analyzed. The Ethics Committee of Shanghai Tongji Hospital approved the present study. Informed consent was obtained from each patient prior to the analyses of the tissues. All cases were confirmed by post-operative pathological diagnosis. Patients who had received neoadjuvant chemotherapy or radiation therapy prior to surgery were excluded from the present study. A total of 20 high-grade ovarian cancer tissue samples (labeled A1-3) and 20 healthy fallopian tube tissue samples (labeled B1-3) were collected.

Cells, cell culture and transfection. Human OVA cell lines (A2780, TOV112D, SKOV3, and OVCAR-3) and the normal ovarian cell line (ISOE80) (from the American Type Culture Collection) were cultured in Dulbecco's modified Eagle's medium (DMEM; Gibco; Thermo Fisher Scientific, Inc.) consisting of $10 \%$ fetal bovine serum (FBS; Gibco; Thermo Fisher Scientific, Inc.) and penicillin in a humidified incubator with $5 \% \mathrm{CO}_{2}$ at $37^{\circ} \mathrm{C}$. Small interfering RNAs (siRNAs) for hsa_circ_0026123 (si-circ0026123; 5'-AAGGAGAGG AATACTAATTATCC-3') (Shanghai GenePharma Co., Ltd.), miR-124-3p mimics (5'-UAAGGCACGCGGUGA AUGCC-3') (Shanghai GenePharma Co., Ltd.), miR-124-3p inhibitors (5'-CGUGUUCACAGCGGACCUUGAU-3'), EZH2 overexpression vector (the sequence was inserted into the pcDNA3.1 vector) (Shanghai GenePharma Co., Ltd.), and their negative controls (Shanghai GenePharma Co., Ltd.) were transfected into cultured SKOV3 cells using Lipofectamine 2000 (Invitrogen; Thermo Fisher Scientific, Inc.) with $50 \mathrm{nM}$ according to standard procedures. Following transfection for $48 \mathrm{~h}, \mathrm{SKOV} 3$ cells were collected for use in the experiments.
To further validate the effects of hsa_circ_0026123 in the in vivo experiments, lentiviral stabilized SKOV3 cells (lentiviral vector: pCDH-CMV-MCS-EF1-copGFP; Novagen) in which hsa_circ_0026123 was silenced were constructed. GFP detection was performed at $72 \mathrm{~h}$ following infection, and when the green fluorescence was $>95 \%$, the transfection was considered successful.

Bioinformatics analysis. The circRNA/miRNA target genes were predicted using Interactome (https://circinteractome. nia.nih.gov/). The interactive association between miR-124-3 and EZH2 was predicted using the Targetscan (http://starbase. sysu.edu.cn/).

Reverse transcription-quantitative PCR (RT-qPCR). Total RNA was extracted from tissues and cells using TRIzol reagent (Invitrogen; Thermo Fisher Scientific, Inc.). The RNA concentration was measured using an ultraviolet spectrophotometer (Hitachi Corporation). Subsequently, the extracted total RNA was reverse transcribed into cDNA using a Reverse Transcription lit (Takara Biotechnology, Inc.). The thermocycling conditions were as follows: $30 \mathrm{sec}$ at $95^{\circ} \mathrm{C}, 5 \mathrm{sec}$ for 40 cycles at $95^{\circ} \mathrm{C}$, and $35 \mathrm{sec}$ at $60^{\circ} \mathrm{C}$. SYBR-Green (Thermo Fisher Scientific, Inc.) was used for qPCR detection. Relative expression was calculated using the $2^{-\Delta \Delta \mathrm{Cq}}$ method (17). GAPDH and U6 were utilized as the internal references. The experiments were repeated 3 times. The primer sequences were as follows: hsa_circ_0026123 forward, 5'-CATCATATC TCAAAGTAAAGTC-3' and reverse, 5'-CCAAGAAGCCCT GAAGACCG-3'; miR-124-3p forward, 5'-ACACTCCAGCTG GGTAAGGCACGCGGTGAA-3' and reverse, 5'-CTCAAC TGGTGTCGTGGAGTCGGCAATTCAGTTGAGGGCATT CAC-3'; GAPDH forward, 5'-TGTTCGTCATGGGTGTGA AC-3' and reverse, 5'-ATGGCATGGACTGTGGTCAT-3'; U6 forward, 5'-CTCGCTTCGGCAGCACA-3' and reverse, 5'-AACGCTTCACGAATTTGCGT-3'.

Western blot analysis. Cells or tissues were resuspended in radioimmunoprecipitation assay (RIPA) lysis buffer (Beyotime Institute of Biotechnology, Inc.) on ice for $30 \mathrm{~min}$. The samples were centrifuged at $15,000 \mathrm{x}$ g for $20 \mathrm{~min}$ at $4^{\circ} \mathrm{C}$, and the supernatants were collected and boiled for $5 \mathrm{~min}$. Protein concentrations were quantified using the Bradford protein assay (Thermo Fisher Scientific, Inc.). Denatured proteins $(15 \mu \mathrm{g})$ were separated by $12 \%$ SDS-PAGE and then electrotransferred onto polyvinylidene difluoride membranes (EMD Millipore). Following blocking with 5\% non-fat milk blocking buffer for $1 \mathrm{~h}$ at room temperature, the membranes were incubated with primary antibodies (all from Abcam) against OCT4 (cat. no. ab200834; 1:500), Nanog (cat. no. ab109250; 1:500) and glyceraldehyde 3-phosphate dehydrogenase (GAPDH) (cat. no. ab8245; $1: 1,000)$ overnight at $4^{\circ} \mathrm{C}$. The membranes were subsequently washed with PBS 3 times and incubated with secondary antibodies (cat. no. ab6728; 1:5,000; Abcam) at room temperature for $1 \mathrm{~h}$. The labeled bands were visualized with an enhanced chemiluminescence reagent using the Bio-Rad Gel Doc 2000 system (Bio-Rad Laboratories, Inc.), and quantified with the QuantityOne software (v4.6; Bio-Rad Laboratories, Inc.). Protein expression levels were normalized to GAPDH. 
Fluorescence in situ hybridization (FISH). Specific probes to hsa_circ_0026123 (Dig-5'-CATTAACATCAAGCTGACCAG TGCACCGG-3'-Dig) were prepared by Geneseed Biotech, Inc. Signals were detected by FITC-conjugated anti-biotin antibodies (Jackson ImmunoResearch, Inc.). Nuclei were counterstained with 4,6-diamidino-2-phenylindole (DAPI; Shanghai Yisheng Biotechnology Co., Ltd.) at room temperature for $15 \mathrm{~min}$. Finally, images were obtained on a Zeiss LSM 700 confocal microscope (Carl Zeiss $\mathrm{GmbH})$.

Cloning formation and cell proliferation assays. A Cell Counting Kit-8 (CCK-8) assay was used to determine cell proliferation. Transfected cells were seeded into 96 -well plates at a density of 2,000 cells/well in triplicate wells. Cell viability was measured using the CCK-8 system (Invitrogen; Thermo Fisher Scientific, Inc.) at 0, 24, 48, 72, and $96 \mathrm{~h}$ after seeding, following standard procedures.

For the colony formation assay, transfected cells were seeded into 6-well plates at a density of 2,000 cells/well and maintained them in DMEM containing 10\% FBS for 10 days. The colonies were imaged with Nikon camera D610 (Nikon Corporation) and counted after fixing with $4 \%$ paraformaldehyde (Shanghai Yisheng Biotechnology Co., Ltd.) and staining with $0.1 \%$ crystal violet (Shanghai Yisheng Biotechnology Co., Ltd.) at room temperature for $15 \mathrm{~min}$.

Cell migration assay. Cell migration was measured using 24-well Transwell ${ }^{\circledR}$ chambers $(8 \mu \mathrm{m}$ pore membrane; BD Biosciences). Cells ( $\left.1 \times 10^{5}\right)$ were plated into the upper chamber with $200 \mu$ l of serum-free medium and the bottom chamber was filled with $500 \mu 1$ DMEM (Gibco; Thermo Fisher Scientific, Inc.) with $20 \%$ FBS (Gibco; Thermo Fisher Scientific, Inc.). Following culture under $37^{\circ} \mathrm{C}$ for 1 day, the cells in the bottom chamber were fixed with $4 \%$ paraformaldehyde for $30 \mathrm{~min}$, and stained them with $0.1 \%$ crystal violet (Shanghai Yisheng Biotechnology Co., Ltd.) for $10 \mathrm{~min}$ at room temperature. The cells were observed and photographed using Axio Observer D1 microscope (magnification, x200; Zeiss AG).

Tumor xenograft formation and metastasis assays. Viable wild-type (WT) or si-circ0026123 SKOV3 cells $\left(2 \times 10^{7}\right)$ (mentioned above) were injected into the right flanks of the nude mice. Tumor sizes were measured every 5 days for 30 consecutive days using a Vernier caliper, and the tumor volume was calculated using the following formula: Volume=length $\mathrm{x}$ width ${ }^{2} \mathrm{x} 0.5$.

Dual luciferase reporter assay. Hsa_circ_0026123-wild type (WT), EZH2 3'-UTR-wild type (WT), hsa_ circ_0026123-mutant (Mut), EZH2 3'-UTR-mutant (Mut) were constructed using pGL3-Basic luciferase vectors (Promega Corporation) and transfected (50 nM) into 239T cells (American Type Culture Collection) with or without NC (50 nM) or miR-124-3p mimics (50 nM), respectively. After 48 h, a dual-luciferase reporter assay kit (Promega Corporation) was used to determine luciferase activity. Renilla luciferase was used for normalization. The luciferase activities were measured using a luciferase assay kit (Promega Corporation). Three independent experiments were performed in triplicate.
Statistical analysis. Differences between groups were assessed using one-way ANOVA method with Tukey's post hoc test. Results were presented as the means \pm SEM. P-values $<0.05$ were considered to indicate statistically significant differences. Statistical analyses were performed using GraphPad Prism 5.02 software (GraphPad, Inc.).

\section{Results}

High hsa_circ_0026123 expression in OVA cells and tissues and its role in migration, proliferation and CSC differentiation. hsa_circ_0026123 expression was detected in 20 OVA tissue samples and corresponding adjacent normal tissues by RT-qPCR. The data revealed that hsa_circ_0026123 expression was significantly increased in OVA tissues when compared with adjacent normal tissues (Fig. 1A), suggesting that hsa_circ_0026123 plays a role in OVA progression. RT-qPCR also revealed that hsa_circ_0026123 expression in human OVA cell lines (A2780, TOV112D, OVCAR-3 and SKOV3) was increased when compared with that in the normal ovarian cell line (ISOE80; Fig. 1B). The SKOV3 cells exhibited the highest hsa_circ_0026123 expression; thus the SKOV3 cells were selected for use in the following experiments. FISH was performed to detect hsa_circ_0026123 subcellular localization. The results revealed that hsa_circ_0026123 was localized in the cytoplasm (Fig. 1C).

To confirm that hsa_circ_0026123 plays a role in the progression of OVA, siRNAs against hsa_circ_0026123 were constructed. The results of RT-qPCR revealed that hsa_circ_0026123 expression in SKOV3 significantly decreased following transfection with siRNA against hsa_circ_0026123 (si-circ0026123) when compared with the negative control (NC; Fig. 2A). CCK-8 (Fig. 2B) and colony formation (Fig. 2C and D) assays revealed that SKOV3 cell proliferation was decreased following the downregulation of hsa_circ_0026123. The results of Transwell assay also demonstrated that the silencing of hsa_circ_0026123 suppressed SKOV3 cell migration (Fig. 2E and F). In addition, western blot analyses revealed that the silencing of hsa_circ_0026123 decreased the protein expression levels of markers (OCT-4 and Nanog) related to CSC differentiation. These results suggested that the downregulation of hsa_circ_0026123 suppressed OVA cell proliferation, migration and CSC differentiation.

miR-124-3p and EZH2 are the downstream targets of

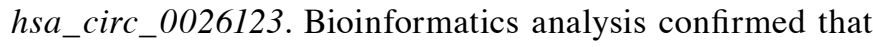
hsa_circ_0026123 targeted miR-124-3p. To further verify this observation, a luciferase reporter vector was constructed. The luciferase reporter assay results revealed that miR-124-3p inhibited luciferase activity following transfection with the WT luciferase reporter vector, while it had no effects on luciferase activity following transfection with a mutated (MUT) luciferase reporter vector, which indicated that miR-124-3p was a target of hsa_circ_0026123 (Fig. 3A and B).

Bioinformatics analysis (http://starbase.sysu.edu.cn/) revealed that a number of genes were the downstream targets of miR-124-3p, including MYLIP, WEE1, BTG2 and EZH2. However, only EZH2 has two binding sites. This indicated that miR-124-3p directly interacted with the EZH2 3'-UTR and suppressed the post-translational EZH2 expression (Fig. 3C). 
A

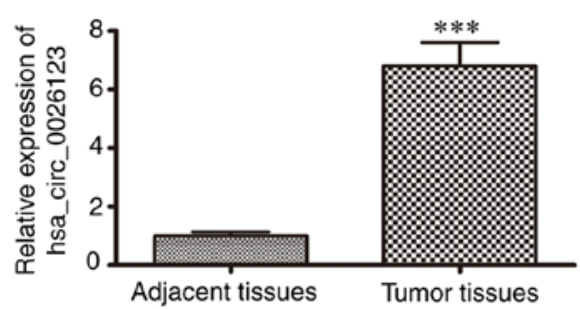

C
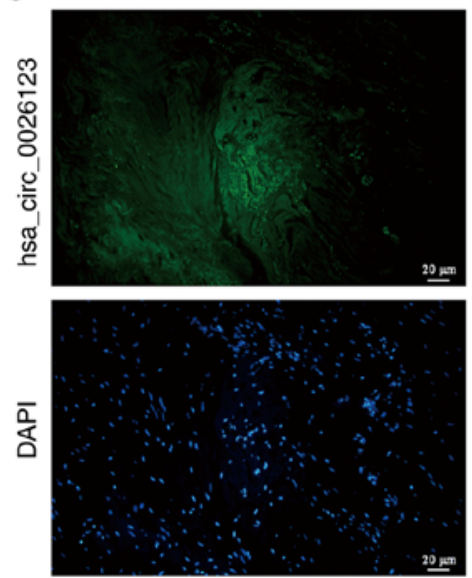

$\mathrm{B}$

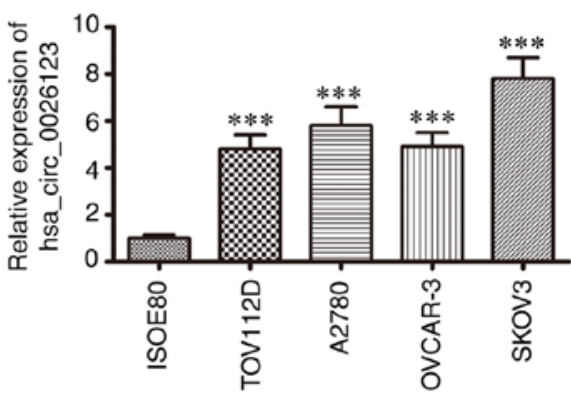

Figure 1. Expression levels of hsa_circ_0026123 are significantly increased in ovarian cancer tissues and cell lines. (A) RT-qPCR revealed that hsa_ circ_0026123 expression was significantly upregulated in ovarian cancer tissues when compared with adjacent tissues. Data are presented as the means \pm SD. ${ }^{* * *} \mathrm{P}<0.001$ vs. adjacent tissues. (B) RT-qPCR revealed the expression of hsa_circ_0026123 in human ovarian cancer cell lines (A2780, TOV112D, OVCAR-3 and SKOV3) and normal ovarian cell line (ISOE80). Data are presented as the means $\pm \mathrm{SD}$. ${ }^{* * * *} \mathrm{P}<0.001$ vs. ISOE80 cells. (C) Fluorescence in situ hybridization was performed to determine the subcellular localization of hsa_circ_0026123. DAPI indicated by nuclear staining (bottom left panel); hsa_circ_0026123 indicated by red fluorescent-tagged hsa_circ_0026123 (top left panel). Merged images are illustrated on the right panel.
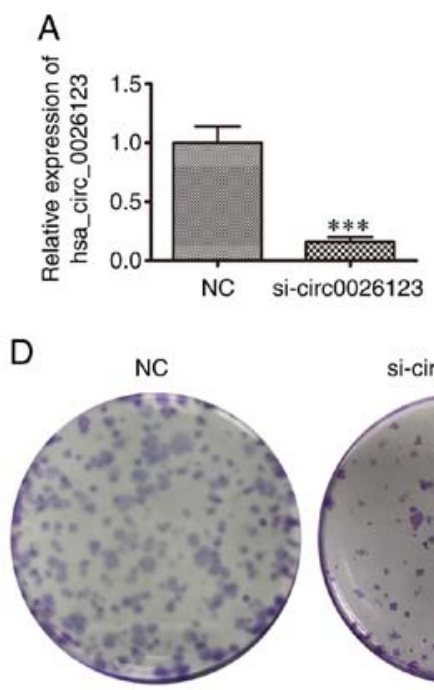

$\mathrm{F}$

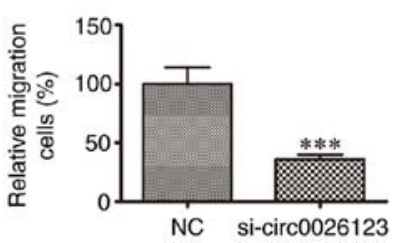

B

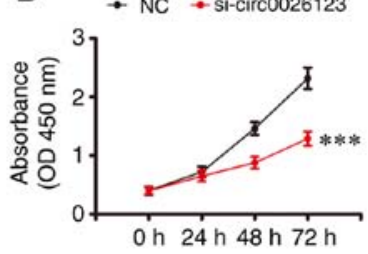

$E$
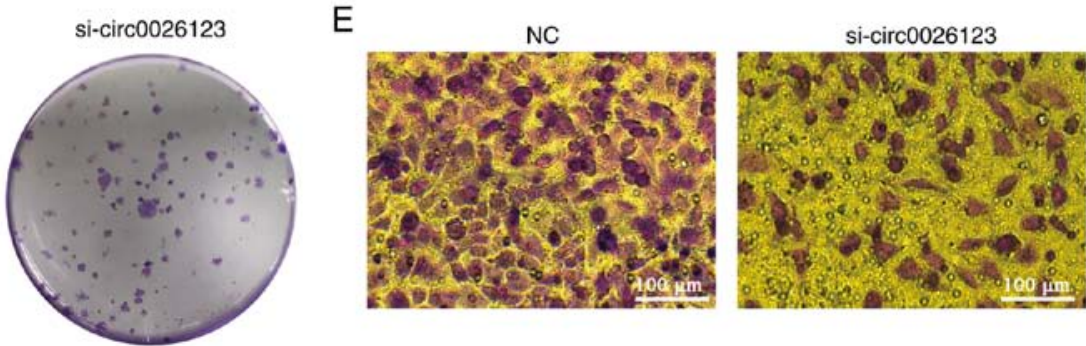

G

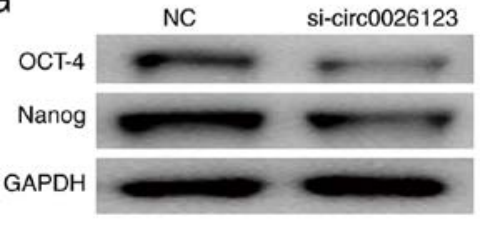

Figure 2. Downregulation of hsa_circ_0026123 suppresses ovarian cancer cell proliferation and migration, and the expression of cancer stem cell (CSC) differentiation-related markers. (A) RT-qPCR revealed the expression of hsa_circ_0026123 in SKOV3 following transfection with siRNA against hsa_circ_0026123 (si-circ0026123) or negative control (NC). Data are presented as the means \pm SD. ${ }^{* * * *} \mathrm{P}<0.001$ vs. NC. (B) CCK-8 assays were performed to assess the proliferation of SKOV3 cells. Data are presented as the means $\pm \mathrm{SD}$. ${ }^{* * *} \mathrm{P}<0.001$ vs. NC. (C and D) Cloning formation assay showing the proliferation of SKOV3 cells. Data are presented as the means \pm SD. ${ }^{* * *} \mathrm{P}<0.001$ vs. NC. (E and F) Cell migration was determined in SKOV3 cells by Transwell ${ }^{\boxplus}$ assays. Data are presented as the means \pm SD. ${ }^{* * *} \mathrm{P}<0.001 \mathrm{vs}$. NC. (G) Western blot analysis of the protein expression of CSC differentiation-related markers. 
A

$\begin{array}{rccc}\text { hsa_circ_0026123-WT } & 5^{\prime} & \begin{array}{c}\text { UGUAUGCCAAGCGUGCCUUU } \\ |:|||||\end{array} \\ \text { miR-124-3p } & 3^{\prime} & \begin{array}{c}\mid \\ \text { CCGUAAGUGGCGCACGGAAU }\end{array} & 5^{\prime} \\ \text { hsa_circ_0026123-Mut } & 5^{\prime} & \text { UGUAUGCCAACGCACGGUUU } & 3^{\prime}\end{array}$

C

$\begin{array}{rrrr}\text { EZH2 3' UTR-WT } & 5^{\prime} & \text {...CUCCUCUGAAACAGCUGCCUUAG... } & 3^{\prime} \\ \text { miR-124-3p } & 3^{\prime} & \text { CCGUAAGUGGCGCACGGAAU } & 5^{\prime} \\ \text { EZH2 3' UTR-Mut } & 5^{\prime} & \text {...CUCCUCUGAAACAGCACGGAAUG.. } & 3^{\prime}\end{array}$

$\mathrm{B}$

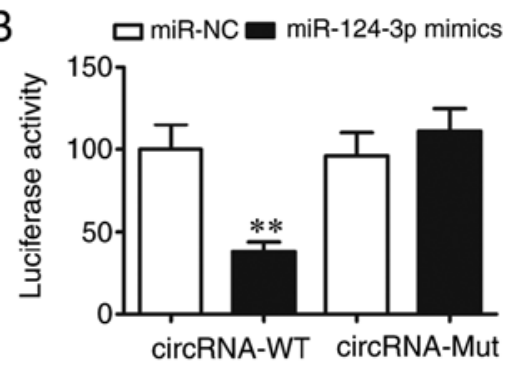

D

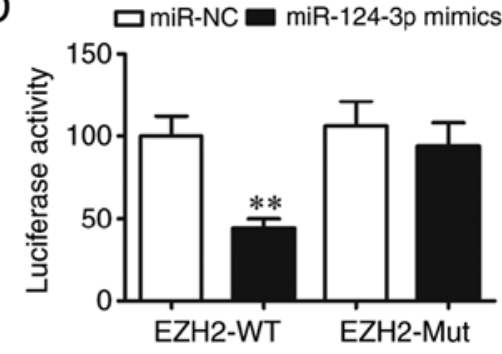

Figure 3. miR-124-3p and EZH2 are the downstream targets of hsa_circ_0026123. (A) Predicted binding sites of miR-124-3p in the hsa_circ_0000291. The mutated (MUT) version of the hsa_circ_0026123 is also shown. (B) Relative luciferase activity was determined $48 \mathrm{~h}$ following transfection with miR-124-3p mimic/normal control (NC) or with the hsa_circ_0026123 wild-type/Mut (WT/MUT) in 293T cells. Data are presented as the means \pm SD. ${ }^{* *} \mathrm{P}<0.01$ vs. NC. (C) Predicted binding sites of miR-124-3p with the 3'-UTR of EZH2. The MUT version of the 3'-UTR-EZH2 is also shown. (D) Relative luciferase activity was determined $48 \mathrm{~h}$ following transfection with miR-124-3p mimic/normal control or with the 3'-UTR-EZH2 WT/MUT in 293T cells. Data are presented as the means $\pm \mathrm{SD} .{ }^{* *} \mathrm{P}<0.01$ vs. NC.

The luciferase reporter assay revealed that miR-124-3p inhibited luciferase activity following transfection with a WT luciferase reporter vector, whereas luciferase activity was not altered after transfection with a MUT luciferase reporter vector, which indicated that EZH2 was the miR-124-3p target (Fig. 3D). Taken together, the results demonstrated that hsa_circ_0026123 knockdown inhibited OVA metastasis and growth by targeting the miR-124-3p/EZH2 axis.

hsa_circ_0026123 knockdown inhibits OVA cell proliferation, migration and CSC differentiation via the regulation of the $m i R-124-3 p / E Z H 2$ axis in vitro. To further explore the regulatory mechanisms, SKOV3 cells were transfected with a hsa_circ_0026123 silencing vector (si-circ0026123), combined with an EZH2 overexpression vector, or were transfected with an miR-124-3p inhibitor. The results confirmed that hsa_circ_0026123 expression was downregulated following transfection with si-circ0026123, as well as by the overexpression of EZH2 or the downregulation of miR-124-3p, which did not 'rescue' hsa_circ_0026123 expression (Fig. 4A). The results RT-qPCR revealed that the downregulation of hsa circ_0026123 promoted miR-124-3p expression. Transfection with the miR-124-3p specific inhibitor suppressed miR-124-3p expression. However, the overexpression of EZH2 did not affect miR-124-3p expression following the silencing of hsa_circ_0026123 in the SKOV3 cell line (Fig. 4B). Western blot analysis confirmed that the downregulation of hsa circ_0026123 suppressed EZH2 expression. The inhibition of miR-124-3p 'rescued' EZH2 expression, and EZH2 expression was increased following transfection with the EZH2 overexpression vector (Fig. 4C). Overall, these results demonstrated that EZH2 was a miR-124-3p downstream target.

The results of CCK-8 (Fig. 4D) and cloning formation (Fig. 4E and F) assays revealed that the overexpression of
EZH2 or the downregulation of miR-124-3p 'rescued' SKOV3 cell proliferation following the silencing of hsa_circ_0026123. The results of Transwell migration assay also revealed that the overexpression of EZH2 or the downregulation of miR-124-3p restored SKOV3 cell migration following the silencing of hsa_circ_0026123 (Fig. 4G and H). Western blot analysis also demonstrated that the overexpression of EZH2 or the downregulation of miR-124-3p restored the expression levels of CSC differentiation-related markers (OCT-4 and Nanog) after the silencing of hsa_circ_0026123, suggesting that hsa_circ_0026123 knockdown inhibited OVA cell migration and proliferation, and decreased the levels of CSC differentiation-related markers via the regulation of the miR-124-3p/ EZH2 axis in vitro.

Overexpression of EZH2 restores cell proliferation and migration, and CSC differentiation following the upregulation of miR-124-3p in vitro. To determine the association between miR-124-3p and EZH2, SKOV3 cells were transfected with miR-124-3p mimics combined with or without the EZH2 overexpression vector. The results of RT-qPCR revealed that miR-124-3p expression was upregulated in SKOV3 cell lines following transfection with miR-124-3p mimic, and EZH2 overexpression had no effect on miR-124-3p expression (Fig. 5A). Western blot analysis revealed that EZH2 expression was downregulated following the overexpression of miR-124-3p, which was restored following transfection with the EZH2 overexpression vector (Fig. 5B). The results of CCK-8 (Fig. 5C) and cloning formation (Fig. 5D and E) assay revealed that cell proliferation was suppressed by miR-124-3p overexpression; however, EZH2 overexpression restored and enhanced the SKOV3 cell proliferation. The results of Transwell migration assays revealed that miR-124-3p expression inhibited cell migration, while EZH2 upregulation 
A

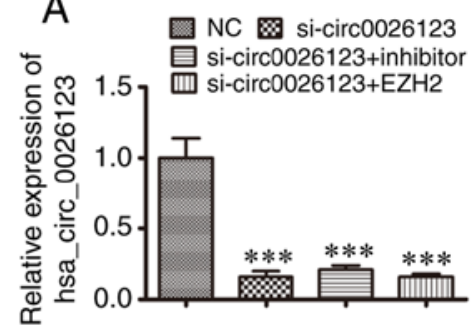

B

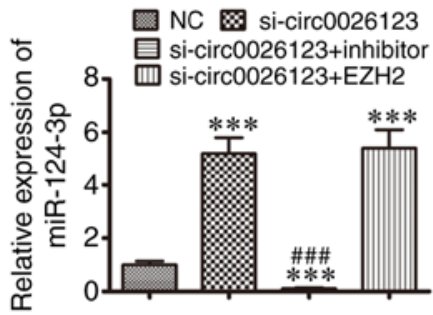

C

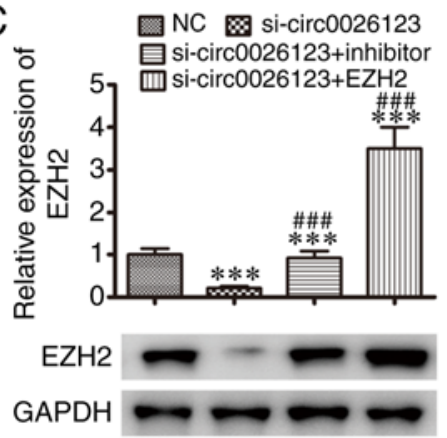

D

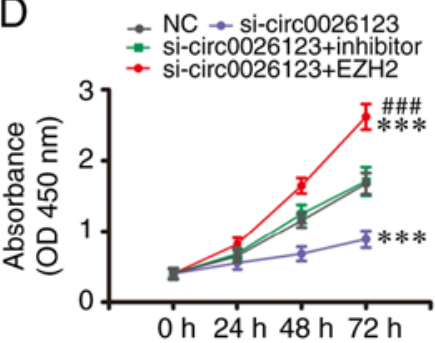

F

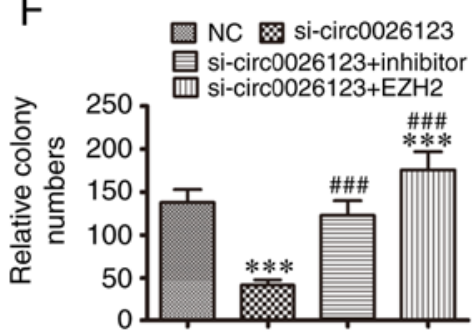

$\mathrm{H}$

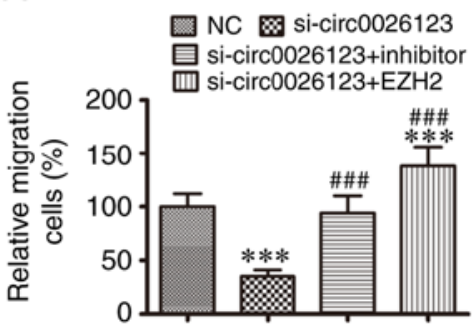

E
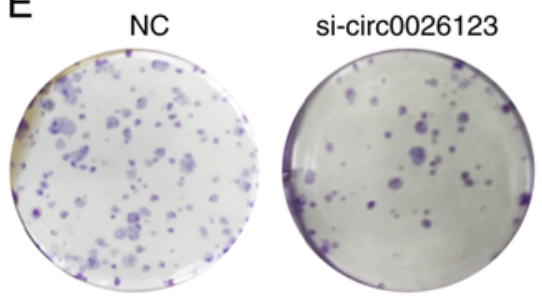

si-circ0026123+ inhibitor
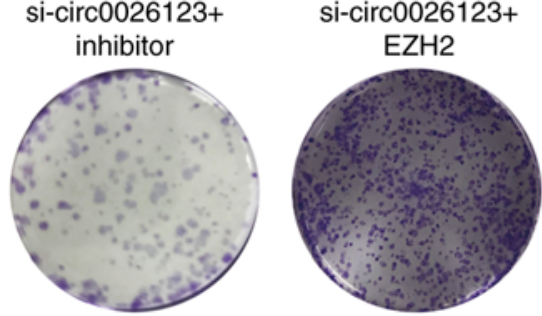

G

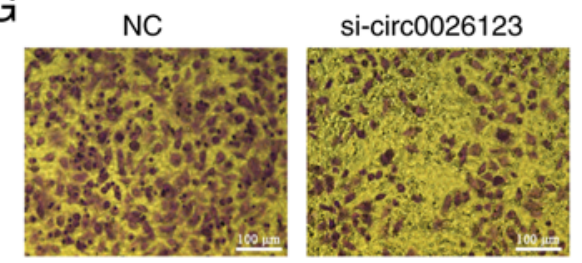

si-circ0026123+ inhibitor si-circ0026123+ EZH2
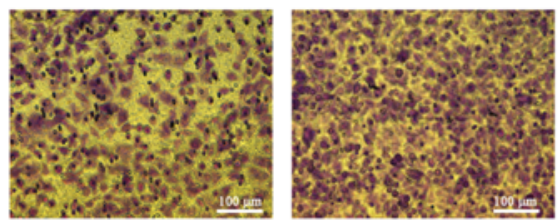

I

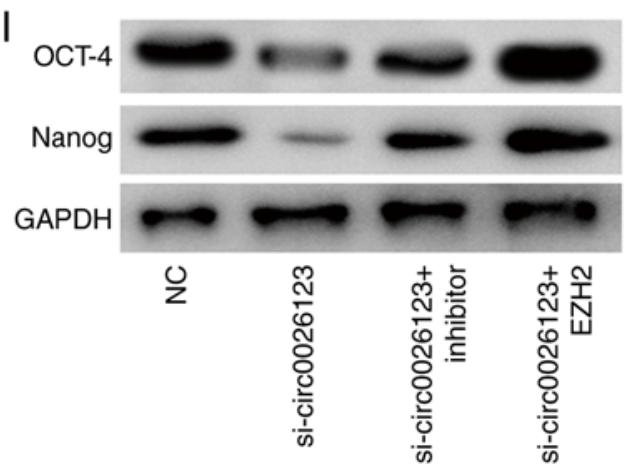

Figure 4. Knockdown of hsa_circ_0026123 inhibits ovarian cancer cell proliferation and migration via the regulation of the miR-124-3p/EZH2 axis in vitro. (A) RT-qPCR assay revealed hsa_circ_0026123 expression in SKOV3 cells following transfection with siRNA against hsa_circ_0026123 combined with or without miR-124-3p inhibitor or the EZH2 overexpression vector. Data are presented as the means \pm SD. ${ }^{* * * *} \mathrm{P}<0.001$ vs. the normal control (NC). (B) RT-qPCR assay showing miR-124-3p expression in SKOV3 cells. Data are presented as the means \pm SD. ${ }^{* * * *} \mathrm{P}<0.001$ vs. NC. ${ }^{\# \# \#<0.001 ~ v s . ~ s i-c i r c 0026123 . ~(C) ~ W e s t e r n ~}$ blots showing EZH2 expression in SKOV3 cells. Relative protein levels were analyzed and data are presented as the means $\pm \mathrm{SD}$. ${ }^{* * * *} \mathrm{P}<0.001$ vs. NC. ${ }^{\# \# "} \mathrm{P}<0.001$ vs. si-circ0026123. (D) CCK-8 assays were performed to assess the proliferation of SKOV3 cells. Data are presented as the means \pm SD. ${ }^{* * *} \mathrm{P}<0.001$ vs. NC. ${ }^{\# \# \# * P}<0.001$ vs. si-circ0026123. (E and F) Colony formation assay showing the proliferation of SKOV3 cells. Data are presented as the means \pm SD. ${ }^{* * *} \mathrm{P}<0.001$ vs. NC. ${ }^{\# \#} \mathrm{P}<0.001$ vs. si-circ0026123. (G and H) Migration of SKOV3 cells was determined by Transwell ${ }^{\circledR}$ assays. Data are presented as the means \pm SD. ${ }^{* * *} \mathrm{P}<0.001$ vs. NC. ${ }^{\# \#} \mathrm{P}<0.001$ vs. si-circ0026123. (I) Western blots showing the relative protein expression levels of markers related to cancer stem cell differentiation. NC, normal control.

increased the migration of SKOV3 cells (Fig. 5F and G). Western blot analysis also demonstrated that miR-124-3p expression suppressed the levels of markers related to CSC differentiation (OCT-4 and Nanog); however, EZH2 overexpression promoted CSC differentiation, as evidenced by the increased levels of OCT-4 and Nanog, even after miR-124-3p overexpression. These results indicated that EZH2 overexpression recovered the growth, migration and the levels of CSC differentiation-related markers following the upregulation of miR-124-3p using in vitro.

hsa_circ_0026123 downregulation suppresses tumor growth in nude mouse xenografts. To confirm whether hsa_circ_0026123 plays a role in OVA progression, lentiviral stable SKOV3 cell strains were constructed in which hsa_circ_0026123 was knocked down. The data indicated that 
A

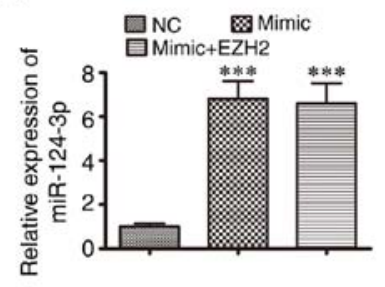

B

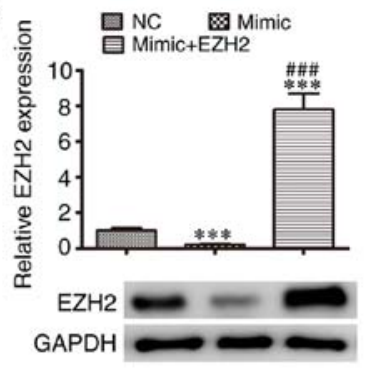

D

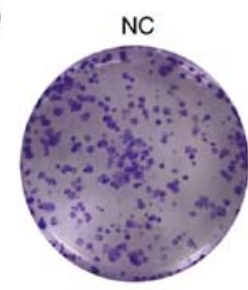

$\mathrm{F}$

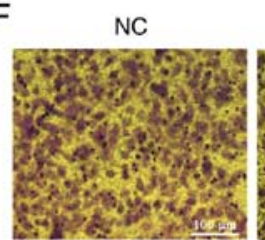

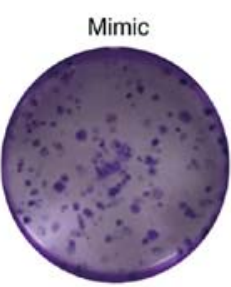

Mimic

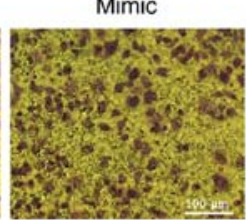

Mimic+EZH2

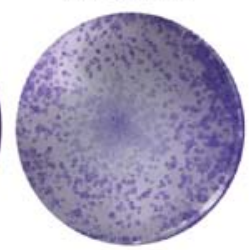

Mimic $+\mathrm{EZH} 2$

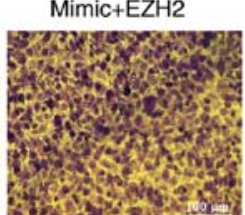

C

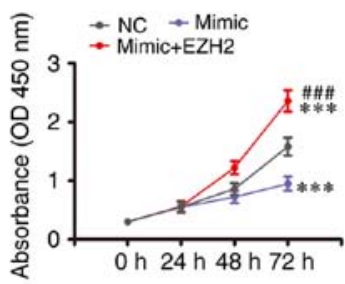

$\mathrm{H}$
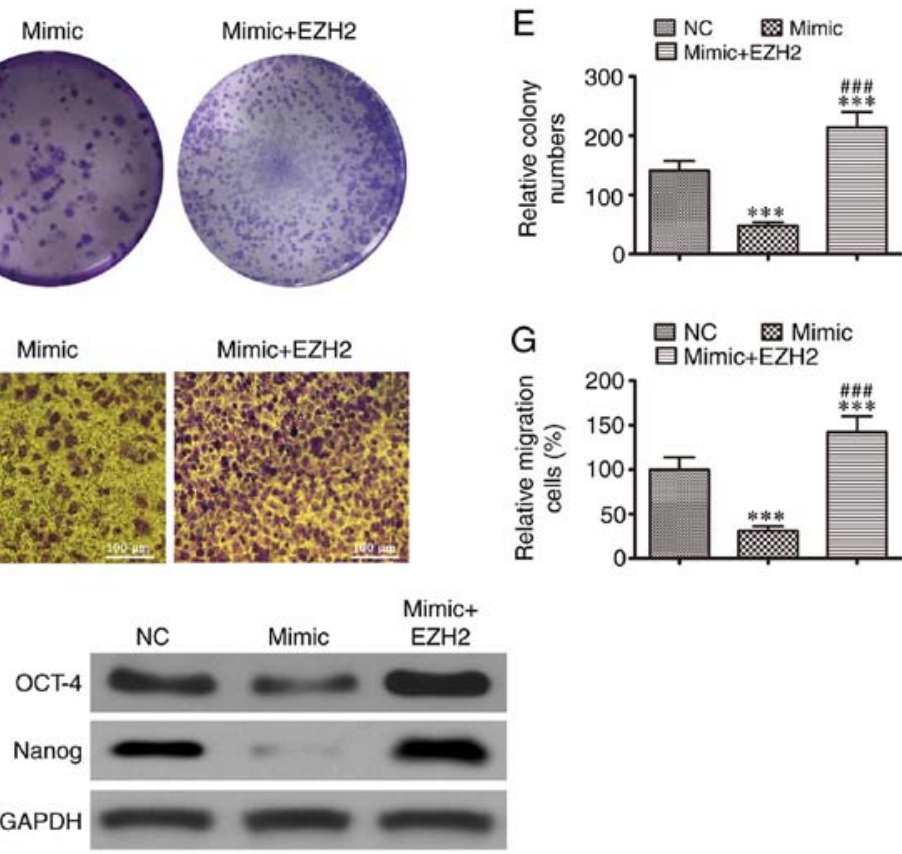

Figure 5. Overexpression of EZH2 restores cell growth and migration following the upregulation miR-124-3p in vitro. SKOV3 cells were transfected with miR-124-3p mimics combined with or without the EZH2 overexpression vector. (A) RT-qPCR assay showing the expression of miR-124-3p in SKOV3 cells. Data are presented as the means \pm SD. ${ }^{* * *} \mathrm{P}<0.001$ vs. NC. (B) Western blot showing EZH2 expression in SKOV3 cells. Relative protein levels were analyzed and data are presented as the means \pm SD. ${ }^{* * *} \mathrm{P}<0.001$ vs. NC. ${ }^{\# \#} \mathrm{P}<0.001$ vs. mimic. (C) CCK8 assays were performed to assess cell proliferation in SKOV3 cells. Data are presented as the mean $\pm \mathrm{SD}$. ${ }^{* * *} \mathrm{P}<0.001$ vs. $\mathrm{NC}$. ${ }^{\# \# /} \mathrm{P}<0.001$ vs. mimic. (D and $\mathrm{E}$ ) Cloning formation assay showing the cell proliferation of SKOV3 cells. Data are presented as the mean $\pm \mathrm{SD}$. ${ }^{* * *} \mathrm{P}<0.001$. ${ }^{\# \# \#} \mathrm{P}<0.001$ vs. mimic. ( $\mathrm{F}$ and $\left.\mathrm{G}\right)$ Cell migration and invasion of SKOV3 cells were determined by Transwell ${ }^{\circledast}$ assays. Data are presented as the means \pm SD. ${ }^{* * *} \mathrm{P}<0.001$ vs. $\mathrm{NC}^{* \# \# \#} \mathrm{P}<0.001$ vs. mimic. (H) Western blots showing the relative protein expression of markers related to cancer stem cell differentiation. NC, normal control.

hsa_circ_0026123 knockdown suppressed the volume and weight of tumors, when compared with the NC group (Fig. 6).

\section{Discussion}

The present study confirmed that hsa_circ_0026123 expression was increased in both OVA tissues and OVA cell lines. hsa_circ_0026123 is 1,155 bp in length and is constructed with part of the TUBA1B gene exon. The downregulation of hsa_circ_0026123 suppressed cell migration and proliferation, and CSC differentiation, suggesting that hsa_circ_0026123 plays a role in OVA progression. Previous studies have reported that circRNA sponges are characterized by increased expression levels and miRNA binding sites, which are likely to be effective sponges, when compared with linear ones $(6,18)$. The sponging activity is the main function of some circRNAs; thus, in tumor development, circRNA/miRNA/mRNA interaction networks may play important roles $(19,20)$.

In the present study, to further identify the downstream miRNA, bioinformatics analysis was used to demonstrated
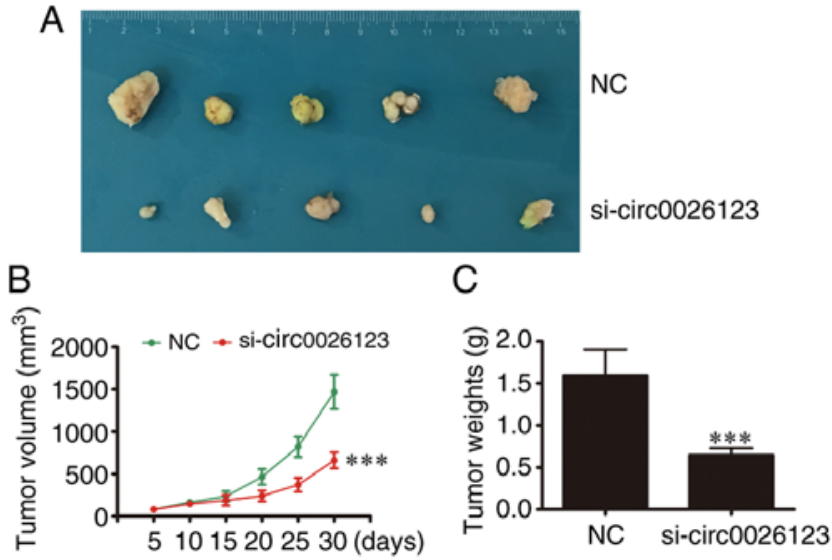

Figure 6. Downregulation of hsa_circ_0026123 suppresses tumor growth in nude mouse xenografts. (A) Representative images of SKOV3 tumor formation in xenografts of nude mice. (B) Summary of tumor volumes in mice that were measured every 5 days. Data are presented as the means \pm SD. ${ }^{* * * *} \mathrm{P}<0.001$ vs. the normal control (NC). (C) Tumor weight was measured after 30 days of injection. Data are presented as the means $\pm \mathrm{SD} .{ }^{* * *} \mathrm{P}<0.001$ vs. NC. 
that miR-124-3p was a target of hsa_circ_0026123. Luciferase reporter assays confirmed that hsa_circ_0026123 interacted with miR-124-3p. The downregulation of hsa_circ_0026123 promoted miR-124-3p expression, and the silencing of miR-124-3p restored the proliferation and migration, and the levels of CSC differentiation-related markers following the silencing of hsa_circ_0026123, suggesting that miR-124-3p exerts an antitumor effect. Previous studies have demonstrated that miR-124-3p upregulation inhibits cancer cell activity, including that of nasopharyngeal carcinoma, bladder cancer, hepatocellular carcinoma, esophageal squamous cell carcinoma and endometrial cancer (21-25). Therefore, hsa circ_0026123 expression promoted the progression of OVA by sponging miR-124-3p.

The present study demonstrated that miR-124-3p interacted with the EZH2 3'-UTR. The results of luciferase reporter assays suggested that miR-124-3p interacted with the 3'-UTR of EZH2. The overexpression of EZH2 restored the proliferation and migration, and the levels of markers related to CSC differentiation following the overexpression of miR-124-3p. It has been reported that the inhibition of EZH2 results in a suppressed epithelial-mesenchymal transition in cancer cells (26). The gene product is a matricellular protein, which stimulates endothelial cell migration and proliferation, as well as the angiogenic activity $(27,28)$. The downregulation of EZH2 suppresses CSC proliferation and differentiation (29-31). Numerous chemical entities have been regarded as EZH2 inhibitors in recent decades, many of which underwent the cancer clinical trials $(32,33)$. The results of the present study demonstrated that hsa_circ_0026123 promoted OVA cell migration and proliferation by sponging miR-124-3p and enhancing EZH2 expression.

In conclusion, the present study provides evidence that hsa_circ_0026123 promotes the proliferation of OVA cells potentially via activating miR-124-3p/EZH2 signaling. The present study suggests that hsa_circ_0026123 is a candidate biomarker for the prognosis and diagnosis of OVA, which extends the drug applications targeting hsa_circ_0026123, suggesting a promising role of hsa_circ_0026123 in the treatment of OVA.

\section{Acknowledgements}

Not applicable.

\section{Funding}

The present study was funded by a grant from the National Natural Science Foundation of China (no. 81702745).

\section{Availability of data and materials}

The datasets used and analyzed during the current study are available from the corresponding author on reasonable request.

\section{Authors' contributions}

$\mathrm{XY}$ and XT designed the research and revised the manuscript. JW and YS performed the experiments and drafted the manuscript. HL performed data analysis. XY and YS revised the manuscript. All authors read and approved the final manuscript.

\section{Ethics approval and consent to participate}

The Ethics Committee of Shanghai Tongji Hospital approved this study. We obtained informed consent from each patient prior to the analyses of tissues. The Ethics Committee of Shanghai Tongji Hospital, Tongji University, Shanghai, China approved all animal experiments.

\section{Patient consent for publication}

Not applicable.

\section{Competing interests}

The authors declare that they have no competing interests.

\section{References}

1. Siegel RL, Miller KD and Jemal A: Cancer statistics, 2018. CA Cancer J Clin 68: 7-30, 2018

2. Rustin G, van der Burg M, Griffin C, Qian W and Swart AM: Early versus delayed treatment of relapsed ovarian cancer. Lancet 377: 380-381, 2011.

3. Doubeni CA, Doubeni AR and Myers AE: Diagnosis and management of ovarian cancer. Am Fam Physician 93: 937-944, 2016.

4. Li Y, Zheng Q, Bao C, Li S, Guo W, Zhao J, Chen D, Gu J, He X and Huang S: Circular RNA is enriched and stable in exosomes: A promising biomarker for cancer diagnosis. Cell Res 25: 981-984, 2015.

5. Zhang SJ, Chen X, Li CP, Li XM, Liu C, Liu BH, Shan K, Jiang Q, Zhao C and Yan B: Identification and characterization of circular RNAs as a new class of putative biomarkers in diabetes retinopathy. Invest Ophthalmol Vis Sci 58: 6500-6509, 2017.

6. Wilusz JE and Sharp PA: Molecular biology. A circuitous route to noncoding RNA. Science 340: 440-441, 2013.

7. Kumar L, Shamsuzzama, Haque R, Baghel T and Nazir A: Circular RNAs: The emerging class of non-coding RNAs and their potential role in human neurodegenerative diseases. Mol Neurobiol 54: 7224-7234, 2017.

8. Piwecka M, Glazar P, Hernandez-Miranda LR, Memczak S, Wolf SA, Rybak-Wolf A, Filipchyk A, Klironomos F, Jara CAC, Fenske P, et al: Loss of a mammalian circular RNA locus causes miRNA deregulation and affects brain function. Science 357: eaam8526, 2017

9. Shan K, Liu C, Liu BH, Chen X, Dong R, Liu X, Zhang YY, Liu B, Zhang SJ, Wang JJ, et al: Circular noncoding RNA HIPK3 mediates retinal vascular dysfunction in diabetes mellitus. Circulation 136: 1629-1642, 2017.

10. Hsiao KY, Lin YC, Gupta SK, Chang N, Yen L, Sun HS and Tsai SJ: Noncoding effects of circular RNA CCDC66 promote colon cancer growth and metastasis. Cancer Res 77: 2339-2350, 2017.

11. Guo Q, He Y, Sun L, Kong C, Cheng Y and Zhang G: In silico detection of potential prognostic circRNAs through a re-annotation strategy in ovarian cancer. Oncol Lett 17: 3677-3686, 2019.

12. Xiong DD, Dang YW, Lin P, Wen DY, He RQ, Luo DZ, Feng ZB and Chen G: A circRNA-miRNA-mRNA network identification for exploring underlying pathogenesis and therapy strategy of hepatocellular carcinoma. J Transl Med 16: 220, 2018.

13. Guan YJ, Ma JY and Song W: Identification of circRNA-miRNA-mRNA regulatory network in gastric cancer by analysis of microarray data. Cancer Cell Int 19: 183, 2019.

14. Zhang L, Zhou Q, Qiu Q, Hou L, Wu M, Li J, Li X, Lu B, Cheng X, Liu P, et al: CircPLEKHM3 acts as a tumor suppressor through regulation of the miR-9/BRCA1/DNAJB6/KLF4/AKT1 axis in ovarian cancer. Mol Cancer 18: 144, 2019.

15. Gao Y, Zhang C, Liu Y and Wang M: Circular RNA profiling reveals circRNA1656 as a novel biomarker in high grade serous ovarian cancer. Biosci Trends 13: 204-211, 2019. 
16. Sheng M, Wei N, Yang HY, Yan M, Zhao QX and Jing LJ: CircRNA UBAP2 promotes the progression of ovarian cancer by sponging microRNA-144. Eur Rev Med Pharmacol Sci 23: 7283-7294, 2019.

17. Livak KJ and Schmittgen TD: Analysis of relative gene expression data using real-time quantitative PCR and the 2(-Delta Delta C(T)) method. Methods 25: 402-408, 2001.

18. Li J, Yang J, Zhou P, Le Y, Zhou C, Wang S, Xu D, Lin HK and Gong Z: Circular RNAs in cancer: Novel insights into origins, properties, functions and implications. Am J Cancer Res 5: 472-480, 2015

19. Liu J, Song S, Lin S, Zhang M, Du Y, Zhang D, Xu W and Wang H: Circ-SERPINE2 promotes the development of gastric carcinoma by sponging miR-375 and modulating YWHAZ. Cell Prolif 52: e12648, 2019.

20. Liu G, Shi H, Deng L, Zheng H, Kong W, Wen X and Bi H: Circular RNA circ-FOXM1 facilitates cell progression as ceRNA to target PPDPF and MACC1 by sponging miR-1304-5p in non-small cell lung cancer. Biochem Biophys Res Commun 513: 207-212, 2019

21. Liu C, Zhang H and Liu H: Long noncoding RNA UCA1 accelerates nasopharyngeal carcinoma cell progression by modulating miR-124-3p/ITGB1 axis. Onco Targets Ther 12: 8455-8466, 2019.

22. Fu W, Wu X, Yang $\mathrm{Z}$ and Mi H: The effect of miR-124-3p on cell proliferation and apoptosis in bladder cancer by targeting EDNRB. Arch Med Sci 15: 1154-1162, 2019.

23. Cui RJ, Fan JL, Lin YC, Pan YJ, Liu C, Wan JH, Wang W, Jiang ZY, Zheng XL, Tang JB and Yu XG: miR-124-3p availability is antagonized by LncRNA-MALAT1 for Slug-induced tumor metastasis in hepatocellular carcinoma. Cancer Med 8: 6358-6369, 2019.

24. Lv Y, Chen S, Wu J, Lin R, Zhou L, Chen G, Chen H and Ke Y: Upregulation of long non-coding RNA OGFRP1 facilitates endometrial cancer by regulating miR-124-3p/SIRT1 axis and by activating PI3K/AKT/GSK-3 $\beta$ pathway. Artif Cells Nanomed Biotechnol 47: 2083-2090, 2019.

25. Zeng B, Zhang X, Zhao J, Wei Z, Zhu H, Fu M, Zou D, Feng Y, Luo H and Lei Y: The role of DNMT1/hsa-miR-124-3p/BCAT1 pathway in regulating growth and invasion of esophageal squamous cell carcinoma. BMC Cancer 19: 609, 2019.
26. Zhao M, Hu X, Xu Y, Wu C, Chen J, Ren Y, Kong L, Sun S, Zhang L, Jin R and Zhou X: Targeting of EZH2 inhibits epithelialmesenchymal transition in head and neck squamous cell carcinoma via regulating the STAT3/VEGFR2 axis. Int J Oncol 55: 1165-1175, 2019.

27. Nishimoto S, Hamajima Y, Toda Y, Toyoda H, Kitamura K and Komurasaki T: Identification of a novel smooth muscle associated protein, smap2, upregulated during neointima formation in a rat carotid endarterectomy model. Biochim Biophys Acta 1576: 225-230, 2002

28. Maier S, Paulsson M and Hartmann U: The widely expressed extracellular matrix protein SMOC-2 promotes keratinocyte attachment and migration. Exp Cell Res 314: 2477-2487, 2008.

29. Liu H, Sun Q, Sun Y, Zhang J, Yuan H, Pang S, Qi X, Wang H, Zhang M, Zhang H, et al: MELK and EZH2 cooperate to regulate medulloblastoma cancer stem-like cell proliferation and differentiation. Mol Cancer Res 15: 1275-1286, 2017.

30. Chen JF, Luo X, Xiang LS, Li HT, Zha L, Li N, He JM, Xie GF, Xie $\mathrm{X}$ and Liang HJ: EZH2 promotes colorectal cancer stem-like cell expansion by activating p21cip1-Wnt/ $\beta$-catenin signaling. Oncotarget 7: 41540-41558, 2016.

31. Karami Madani G, Rad A, Molavi M, Ardalan Khales S, Abbaszadegan MR and Forghanifard MM: Predicting the correlation of EZH2 and cancer stem cell markers in esophageal squamous cell carcinoma. J Gastrointest Cancer 49: 437-441, 2018

32. Zhang H, Qi J, Reyes JM, Li L, Rao PK, Li F, Lin CY, Perry JA, Lawlor MA, Federation A, et al: Oncogenic deregulation of EZH2 as an opportunity for targeted therapy in lung cancer. Cancer Discov 6: 1006-1021, 2016.

33. Stazi G, Zwergel C, Mai A and Valente S: EZH2 inhibitors: A patent review (2014-2016). Expert Opin Ther Pat 27: 797-813, 2017.

This work is licensed under a Creative Commons Attribution-NonCommercial-NoDerivatives 4.0 International (CC BY-NC-ND 4.0) License. 